\title{
Differential Display
}

National Cancer Institute

\section{Source}

National Cancer Institute. Differential Display. NCI Thesaurus. Code C18483.

Variation of the polymerase chain reaction used to identify differentially expressed genes.

mRNA from two different tissue samples is reverse transcribed, then amplified using short, intentionally nonspecific primers. The array of bands obtained from a series of such amplifications is run on a high resolution gel, and the array of bands compared with analogous arrays from different samples. Any bands unique to single samples are considered to be differentially expressed; they can be purified from the gel, and sequenced and used to clone the full-leng th cDNA. Similar in result to subtractive hybridization. (DCB-CB) 